\title{
Extraintestinal manifestations were common in children with celiac disease and were more prevalent in patients with more severe clinical and histological presentation
}

Samuli Nurminen ${ }^{1,2}$, Laura Kivelä ${ }^{2}$, Heini Huhtala ${ }^{3}$, Katri Kaukinen ${ }^{1,4}$, Kalle Kurppa ${ }^{1,2}$

Affiliations: ${ }^{1}$ Faculty of Medicine and Life Sciences, University of Tampere, Finland;

${ }^{2}$ Tampere Center for Child Health Research, University of Tampere and Tampere University

Hospital, Tampere, Finland; ${ }^{3}$ Faculty of Social Sciences, University of Tampere, Tampere,

Finland; ${ }^{4}$ Department of Internal Medicine, Tampere University Hospital, Finland

Short title: Extraintestinal manifestations in paediatric celiac disease

Corresponding author: Kalle Kurppa, M.D, PhD, Faculty of Medicine and Life Sciences,

University of Tampere, FIN-33014, Tampere, Finland.

E-mail: kalle.kurppa@uta.fi

Telephone number: +358 33551 8403; Fax number: +358 335518402

\section{Conflicts of Interest}

The authors have no conflicts of interest to disclose

\section{Funding}

This study received external support from the Academy of Finland, the Sigrid Juselius

Foundation, the Mary and Georg Ehrnrooth Foundation, the Foundation for Pediatric

Research and the Finnish Medical Foundation 


\section{ABSTRACT}

Aim: This study investigated the prevalence of extraintestinal manifestations (EIM) in paediatric celiac disease and their associations with other disease features.

Methods: Researchers at the University of Tampere, Finland, compared EIM in 511 children diagnosed with celiac disease from 2003-2014 and 180 diagnosed with functional gastrointestinal disorders from 2007-2013. Disease severity and dietary responses were also compared between celiac children diagnosed by screening $(n=146)$ or because of EIM $(n=116)$ or gastrointestinal $(n=249)$ symptoms.

Results: Celiac patients had more EIM (62\%) than those with functional disorders (33\%). The most common EIM in celiac children were poor growth (27\%) and anaemia (18\%). Children with celiac disease often showed fatigue (8\%) and symptoms affecting the skin (15\%), nervous system (9\%) and joints (6\%). Celiac patients with EIM as their main clinical presentation had more severe symptoms and histological damage at diagnosis than those with gastrointestinal presentation and screen-detected cases. The subgroups did not differ with regard to other clinical and laboratory parameters and dietary adherence. Concomitant EIM were also common in children diagnosed because of gastrointestinal presentation (60\%) and by screening (37\%).

Conclusion: EIM were common in celiac disease and associated with more severe clinical and histological presentation.

Keywords: Celia disease, Extraintestinal manifestations, Histology, Paediatric, Serology, 


\section{Key notes}

- This study investigated the prevalence of extraintestinal manifestations (EIM) and their association with other disease features in paediatric celiac disease.

- We found that EIM were common in children with untreated celiac disease and their presence was associated with more severe clinical and histological presentation.

- In addition, the 511 children with celiac disease had higher levels of EIM than the 180 children with functional gastrointestinal disorders we studied. 


\section{INTRODUCTION}

The classical clinical presentation of celiac disease is early-onset diarrhoea and malabsorption, with other common gastrointestinal symptoms being abdominal pain, bloating, nausea and constipation. Despite this, patients may also present with a variety of extraintestinal manifestations (EIM), for example poor growth, increased liver enzymes, dermatitis herpetiformis or other rashes, arthritis and various neurological symptoms (1-3). It has been suggested that these atypical symptoms may occur even more frequently than the classical gastrointestinal presentation $(1,2,4)$.

Although many EIM of celiac disease have been known for some time (5), their actual prevalence is poorly defined. It is even more unclear whether the presence of such symptoms affects the severity of histological damage and other characteristics of celiac disease at the time of diagnosis. The great majority of celiac disease sufferers currently remain either unrecognised or there is an unacceptably long diagnostic delay (6). A better understanding of the heterogeneous EIM would increase diagnostic efficacy and prevent the unnecessary burden, and possibly the severe complications, associated with unrecognised celiac disease.

The aim of this study was to investigate the prevalence of EIM symptoms in children with celiac disease. In order to benchmark the observed prevalence, they were compared with children suffering from functional gastrointestinal symptoms. In addition, we sought to establish whether the main clinical presentation of celiac disease, namely extraintestinal symptoms versus gastrointestinal symptoms and screen-detected symptoms, affected other disease features and the patients' responses to a gluten-free diet. 


\section{PATIENTS AND METHODS}

\section{Study subjects and design}

The study was carried out in Finland, at the Center for Child Health Research, University of Tampere and Tampere University Hospital, and in the Department of Pediatric

Gastroenterology of Tampere University Hospital. We included all children under the age of 18 diagnosed with celiac disease from 2003-2014 or functional gastrointestinal disorder from 2007-2013. Children with an uncertain diagnosis or potential celiac disease were excluded from further analyses. Medical information was collected from the hospital patient records and from our regularly updated paediatric research database, which has previously been described (4). From 2012 onwards the celiac disease patients were enrolled as part of a prospective patient collection, but all the other study data were collected retrospectively.

The diagnosis of celiac disease was based on the demonstration of small-bowel mucosal villous atrophy and crypt hyperplasia (7). Functional gastrointestinal disorder was diagnosed in children who had undergone meticulous clinical and laboratory investigations and gastrointestinal endoscopy. These included intestinal biopsies because of undefined abdominal symptoms, such as pain, dyspepsia and nausea, without findings suggestive of any organic disease (8).

Clinical information, including the presence of EIM at diagnosis as reported by the attending physician, was collected on all the children. Celiac patients also underwent analyses of the disease-specific transglutaminase 2 and endomysial antibodies and other relevant laboratory parameters, as described below. Furthermore, the presence of concomitant associated conditions such as type 1 diabetes mellitus, autoimmune thyroidal disease and Down syndrome were noted. After these analyses, children with celiac disease were further divided into those diagnosed because of extraintestinal or gastrointestinal manifestations 
symptoms and those who were detected during at-risk group screening. The groupings were carried out on the basis of the original notes in the medical record. These three subgroups were compared in terms of clinical characteristics, histology and serology and adherence and responses to a gluten-free diet.

The collection of patient information from the medical records was approved by the Department of Pediatrics, Tampere University Hospital. In addition, prospective patient enrolment and data gathering were accepted by the Ethics Committee of the Pirkanmaa Hospital District. All participating children and, or, their parents provided written informed consent, depending on the patient's age.

\section{Presence of EIM}

The possible presence of EIM was recorded for all study children. These features included skin symptoms, such as dermatitis herpetiformis, atopic dermatitis, erythema nodusum, acanthosis nigricans or an unspecified rash; anaemia; poor growth and dental enamel defects and neurological symptoms, such as migraine or other headaches. They also included worsening of epilepsy or gluten ataxia; arthritis or other joint symptoms; recurrent aphtous ulcers and other mouth symptoms, hypertransaminasemia, defined as alanine aminotransferase > $40 \mathrm{U} / \mathrm{l}$; fatigue; eye symptoms, such as episcleritis or uveitis, and other celiac disease associated extraintestinal symptoms as described in the literature $(1,2,9)$. Anaemia was defined based on age and gender-specific reference values, as previously described in detail (10). Poor growth was defined as an abnormal deceleration of growth compared with age and gender-specific reference charts or growth below the expected target height, as previously described in detail (3). This definition is meant to be a sensitive screening tool for early detection of possibly treatable problems and does not necessary mean 
that the child will have abnormal growth.

The severity of symptoms at diagnosis was determined on basis of the clinical picture as reported by the clinician. Mild was occasional disturbing symptoms, moderate was symptoms that were more distracting or frequent and severe symptoms were those that seriously disturbed daily life.

\section{Celiac disease serology and laboratory parameters}

Serum transglutaminase 2 antibodies were measured using an conventional ELISA (Phadia AB, Uppsala, Sweden) before 2011 or an automatised human recombinant-based EliA assay (Phadia AB, Uppsala, Sweden) from 2011 onwards. In our laboratory a transglutaminase 2 value of $\geq 7 \mathrm{U} / \mathrm{l}$ is considered positive and the maximum reported value is $120 \mathrm{U} / \mathrm{l}$. Serum endomysial were measured by indirect immunofluorescence using human umbilical cord as a substrate, as previously described (11) An endomysial dilution of $1: \geq 5$ was considered positive and positive sera were further diluted from 1:50 to 1:4000 until negative.

The following laboratory values measured from blood samples at the time of celiac disease diagnosis were collected from each child when available: haemoglobin (g/l), mean corpuscular volume (fl), total iron ( $\mu \mathrm{mol} / \mathrm{l})$, alanine aminotransferase $(\mathrm{U} / \mathrm{l})$, ferritin ( $\mu \mathrm{g} / \mathrm{l})$, transferrin receptor $1(\mathrm{mg} / \mathrm{l})$, alkaline phosphatase $(\mathrm{U} / \mathrm{l})$, albumin $(\mathrm{g} / \mathrm{l})$, thyroidstimulating hormone (mU/l) and thyroxin (pmol/l).

\section{Small-bowel mucosal morphology}

At least four forceps biopsy samples were taken from the distal duodenal mucosa and from the duodenal bulb from 2012 onwards in all cases were celiac disease was suspected. The specimens were further processed and analysed in the hospital pathology unit. Only correctly 
oriented specimens were accepted for further microscopic analyses. If there was poor orientation, new cuttings were requested according to our standard operating procedures. In the present study the severity of the mucosal damage was categorised, based on the hospital pathologist's original grading, into partial, subtotal and total villous atrophy.

\section{Adherence and responses to a gluten free diet}

All celiac disease patients were placed on a gluten-free diet within 1-4 weeks of diagnosis, under the supervision of a qualified dietician. Adherence to the diet were categorised as strict diet, occasional lapses and no diet and evaluated during follow-up visits, based on family interviews and the results of serology, as previously described (14). The patient's response to the diet was also assessed and classified. A response was defined as the alleviation of symptoms and normalised or constantly decreasing celiac autoantibody levels and no response was defined as persistent symptoms and, or, positive autoantibodies.

\section{Statistical analysis}

All statistical analyses were performed using SPSS for Windows, version 21.0 (IBM, New York, USA. Differences between the groups were compared by the Mann-Whitney U test, the Kruskal-Wallis test, Pearson’s chi-square and Fisher's exact test. Data are expressed as either medians with upper and lower quartiles or as percentages. A p value of $<0.05$ was considered significant.

\section{RESULTS}

The final study cohort comprised 511 children with celiac disease and 180 with functional gastrointestinal disorders. The celiac patients were younger than those with functional gastrointestinal disorders, with a median age of 7.6 (4.8-11.6 years) versus 10.6 (6.8-13.7) 
years, and they were more likely to be girls (65 versus 53\%) (Table 1). EIM were present almost twice as often in celiac disease than functional disorders (62 versus 33\%) (Table 1). The most common EIM in celiac disease were poor growth (27\%), anaemia (18\%) and skin symptoms (15\%) and other frequent presentations were hypertransaminasemia (9\%), neurological symptoms (9\%), fatigue (8\%) and joint symptoms (6\%). Dermatitis herpetiformis, a well-known skin manifestation of celiac disease, was found in eight (1.6\%) children with celiac disease. Recurrent aphtous ulcers were rare in both groups and dental enamel defects were found only in three children with celiac disease. A variety of other EIM were reported in celiac disease patients (Table 1).

The main reason for suspected celiac disease was EIM in 116 (23\%) patients and gastrointestinal presentation in 249 (49\%) patients. A further 146 (29\%) patients were detected during screening of high-risk groups. The most common indications for screening were a family risk of celiac disease (42\%) and previously diagnosed type 1 diabetes (12\%). Concomitant EIM were also common in the two latter groups and these patients displayed particularly dermatological and neurological symptoms, poor growth, fatigue, anaemia and hypertransaminasemia (Table 2).

We also analysed the children with celiac disease based on whether they were diagnosed by EIM, gastrointestinal symptoms or screening. This showed that with children with EIM as the main presentation had more severe villous atrophy than those diagnosed by screening or gastrointestinal symptoms (Fig 1a), while screen-detected children had the highest levels of haemoglobin, albumin and transglutaminase 2 (Table 3). There was also a clinically small, but statistically significant $(\mathrm{p}=0.032)$, difference in the median haemoglobin values between the three sub-groups (123, 24 and $126 \mathrm{~g} / \mathrm{l}$, respectively). The three subgroups did not differ with regard to other laboratory values, growth parameters and age (Table 3), gender distribution (girls 64\%, 67\% and 62\%, p=0.550) and prevalence of celiac disease- 
associated conditions $(10 \%, 10 \%$ and $17 \%, \mathrm{p}=0.114)$. The severity of symptoms prior to diagnosis was considered mild in $22 \%$ and moderate or severe in $78 \%$ of children with EIM presentation and the corresponding figures in those with gastrointestinal presentation was $53 \%$ and $47 \%(\mathrm{p}<0.001)$. In addition, $45 \%$ of the screen-detected children reported mild and 14\% moderate symptoms.

Children in all three celiac disease subgroups showed excellent and practically equal adherence to the gluten-free diet (Fig 1b). Likewise, the beneficial response to dietary treatment was comparable between the groups: EIM 98\%, gastrointestinal 96\% and screendetected $97 \%(\mathrm{p}=0.556)$.

\section{DISCUSSION}

There were two main findings in the present study. First, we observed that EIM were clearly overrepresented in children with untreated celiac disease compared to children with functional gastrointestinal disorders. Second, the presence of EIM at celiac disease diagnosis was associated with a more severe clinical and histological presentation.

We found that particularly common EIM in children with celiac disease were poor growth, anaemia and skin symptoms. In fact, poor growth was noted in almost one-third of the celiac patients at diagnosis. This was in line with the findings of Jericho et al (12), who reported a prevalence of 33\% for growth failure in paediatric celiac disease. On the other hand, markedly lower (13-15) and higher (16) frequencies have been reported by other studies. Apart from differences in study design and population, the inconsistent results might be, at least partly, explained by variable definitions. We defined poor growth using specific Finnish growth references, which have all been well validated and may even lead to improved celiac disease diagnostics (17). However, these screening charts are more sensitive for growth 
variations than the more widely used World Health Organization criteria, which explains the unusually high number of children with abnormal growth in the functional gastrointestinal disorder group. This may also complicate comparisons with previous studies. Poor growth was classified here as an EIM because it might also be caused by other pathophysiological mechanisms than just malabsorption (2).

The second most common EIM in celiac disease was anaemia. Although the incidence was about as common as observed in our other recent studies $(4,10)$, the prevalence of anaemia was similar to poor growth in that it was markedly lower in this study than in most previous reports $(13,15,18)$. One explanation could be that, overall, nutritional status is good in Finnish children and anaemia and iron deficiency are rare. Moreover, the presence of anaemia at the time of celiac disease diagnosis is known to be associated with a severe clinical and histological presentation and long diagnostic delay $(10,18)$ and these are both less frequent in Finland than in many other countries (4). Similarly as with growth failure, the definition of anaemia as an EIM can be debated, but the concept is based on evidence that anaemia in celiac disease is not necessarily associated with the degree of intestinal malabsorption $(2,10,19)$. Besides iron deficiency, it can also be caused factors such as chronic inflammation and bone marrow suppression (20). In general, the pathophysiology of EIM in celiac disease is poorly known, but there are probably several mechanisms, such as chronic inflammation, nutritional deficiencies and hormonal changes (2). Furthermore, autoantibodies targeted, for example, against the brain in gluten ataxia (21) and the bones in osteoporosis (22) may not be just surrogate markers, but may also play a role in the pathogenesis.

Skin manifestations were another common finding in untreated celiac disease. We found that eight (1.6\%) children had dermatitis herpetiformis, a well-known EIM of celiac disease, whereas the association between other rashes and celiac disease is less clear. 
Unspecific skin symptoms, such as viral exanthemas and atopic eczema, are rather common in children, but the overrepresentation of rashes in celiac children compared to those with functional symptoms in this study suggests that these were indeed related to celiac disease. The relative rarity of dermatitis herpetiformis in the children in our study was in accord with the latest evidence (23). There have been a dearth of studies regarding the prevalence of other rashes in celiac disease patients, but two papers have reported figures comparable to ours $(12,14)$.

Of the other EIM, neurological and joint symptoms, fatigue and hypertransaminasemia were particularly frequent in our celiac disease patients. The prevalence of neurological symptoms was consistent with findings from Finland and Italy $(13,15)$ and they were reported in up to $51 \%$ of Israeli children with celiac disease $(14)$. Fatigue and joint symptoms were more common in children with celiac disease than in those with functional disorders, but less common than reported by other authors $(18,24,25)$. In our earlier study, $15 \%$ of children with untreated celiac disease had elevated alanine aminotransferase values (26) and even higher rates have been reported (27). Nevertheless, this study used a higher cut-off for increased values than our previous study, since mild hypertransaminasemia usually has no major clinical significance (26). In contrast to the above-mentioned symptoms, mouth and teeth findings were quite rare. The prevalence of enamel defects and aphtous ulcers has also varied substantially in other studies, probably due to their vague nature and difficult definition $(13,28)$. Altogether, the marked variations between studies in the prevalence of extraintestinal symptoms emphasises the need for prospective studies.

We found that children with EIM had more severe duodenal lesions than those with gastrointestinal presentation. The histology has not previously been studied in these circumstances, but Mubarak et al (18) reported that EIM were more frequent in subjects with 
high transglutaminase 2 values. The surprising presence of the highest antibody levels in the screen-detected children might have been due to clinicians' reluctance to perform gastroscopy in these often asymptomatic patients with low positive serology. In any case, the association between clinical and histologic presentation in celiac disease indicates a pathogenic link. In inflammatory bowel disease, for instance, antigen cross-reactivity, malabsorption and certain human leukocyte antigen phenotypes are thought to be associated with the presence of EIM (29), while there a is lack of similar studies in celiac disease. Aside from the previously mentioned factors in inflammatory bowel disease, the relationship between intestinal microbiota and EIM is particularly interesting (30). Even though EIM associated with more severe presentation in both conditions, celiac disease patients generally have a general better prognosis since the pathogenic process can be reversed by specific treatment.

The main strengths of the present study were the large cohorts of representative celiac disease patients and controls and the wide range of clinically relevant data available on each child. We recognise that there were also several limitations. The main limitation was the retrospective design and lack of systematic questionnaires for the evaluation of symptoms. This might have led, for example, to us underestimating mild or vague symptoms such as enamel defects, aphtous stomatitis and fatigue. Retrospective grouping of the study children to those with EIM or gastrointestinal presentation was challenging and inevitably subjective, particularly if a patient was suffering from more than one symptom simultaneously. The lack of prospective data might also have affected the assessment of dietary responses, as physicians may have focused mostly on alleviating classical gastrointestinal symptoms. Moreover, we were not able to find out whether the symptoms disappeared totally when the children were on a gluten-free diet, which would have further supported the causal relationship between EIM and celiac disease. The retrospective design led to missing growth data and laboratory values from some of the patients, which may have caused selection bias. 
We also were not able to evaluate the exact diagnostic latency in celiac disease. This would have been important, as it is possible that the frequency of EIM was affected by the duration of untreated disease. In addition, the possibility that asymptomatic children with low positive serology were less frequently biopsied might have biased the differences between screendetected and symptomatic patients. Finally, the lack of a standardised international definition of extraintestinal symptoms makes comparing our data with other studies challenging.

\section{CONCLUSION}

We found that EIM were common in children with celiac disease and were associated with a more severe clinical and histological presentation. It is, therefore, important that clinicians consider the possibility of celiac disease as a cause for variable EIM and to specifically consider their presence in newly diagnosed celiac patients. In the future, large multi-centre prospective studies would provide even more robust information on the prevalence of EIM in celiac disease.

Abbreviations

EIM, extraintestinal manifestations 


\section{References}

1. Hernandez L, Green PH. Extraintestinal manifestations of celiac disease. Curr Gastroenterol Rep 2006; 8: 383-9

2. Leffler DA, Green PH, Fasano A. Extraintestinal manifestations of coeliac disease. Nat Rev Gastroenterol Hepatol 2015; 12: 561-71

3. Nurminen S, Kivelä L, Taavela J, Huhtala H, Mäki M, Kaukinen K, et al. Factors associated with growth disturbance at celiac disease diagnosis in children: A retrospective cohort study. BMC Gastroenterol 2015; 15: 125

4. Kivelä L, Kaukinen K, Lähdeaho ML, Huhtala H, Ashorn M, Ruuska T, et al. Presentation of celiac disease in Finnish children is no longer changing: a 50-year perspective. J Pediatr 2015; 167: 1109-15

5. Lindberg T, Berg NO, Borulf S, Jakobsson I. Liver damage in coeliac disease or other food intolerance in childhood. Lancet 1978; 1: 390-1

6. Fuchs V, Kurppa K, Huhtala H, Collin P, Mäki M, Kaukinen K. Factors associated with long diagnostic delay in celiac disease. Scand J Gastroenterol 2014; 49: 1304-10

7. Walker-Smith J, Guandalini S, Schmitz J, DH Shmerling, JK Visakorpi. Revised criteria for diagnosis of coeliac disease. Report of Working Group of European Society of Paediatric Gastroenterology and Nutrition. Arch Dis Child 1990; 65: 90911

8. Hyams JS, Di Lorenzo C, Saps M, Shulman RJ, Staiano A, van Tilburg M. Functional disorders: children and adolescents. Gastroenterology 2016; 150: 1456-68

9. Zingone F, Swift GL, Card TR, Sanders DS, Ludvigsson JF, Bai JC. Psychological morbidity of celiac disease: A review of the literature. United European Gastroenterol J 2015; 3: 136-45

10. Rajalahti T, Repo M, Kivelä L, Huhtala H, Mäki M, Kaukinen K, et al. Anemia in 
pediatric celiac disease: association with clinical and histological features and response to gluten-free diet. J Pediatr Gastroenterol Nutr 2017; 64: e1-e6

11. Kurppa K, Paavola A, Collin P, Sievänen H, Laurila K, Huhtala H, et al. Benefits of a gluten-free diet for asymptomatic patients with serologic markers of celiac disease. Gastroenterology 2014; 147: 610-617

12. Jericho H, Sansotta N, Guandalini S. Extra-intestinal manifestations of celiac disease: effectiveness of the gluten free diet. J Pediatr Gastroenterol Nutr 2017; 65: 75-7.

13. Bottaro G, Cataldo F, Rotolo N, Spina M, Corazza GR. The clinical pattern of subclinical/silent celiac disease: an analysis on 1026 consecutive cases. Am J Gastroenterol 1999; 94: 691-6

14. Zelnik N, Pacht A, Obeid R, Lerner A. Range of neurologic disorders in patients with celiac disease. Pediatrics 2004; 113: 1672-6

15. Savilahti E, Kolho KL, Westerholm-Ormio M, Verkasalo M. Clinics of coeliac disease in children in the 2000s. Acta Paediatr 2010; 99: 1026-1030

16. Rashid M, Cranney A, Zarkadas M, Graham ID, Switzer C, Case S, et al. Celiac disease: evaluation of the diagnosis and dietary compliance in Canadian children. Pediatrics 2005; 116: e754-9

17. Saari A, Harju S, Mäkitie O, Saha MT, Dunkel L, Sankilampi U. Systematic growth monitoring for the early detection of celiac disease in children. JAMA Pediatr 2015; 169: e1525

18. Mubarak A, Spierings E, Wolters VM, Otten HG, ten Kate FJ, Houwen RH. Children with celiac disease and high tTGA are genetically and phenotypically different. World J Gastroenterol 2013; 19: 7114-20

19. Repo M, Lindfors K, Mäki M, Huhtala H, Laurila K, Lähdeaho ML, et al. Anemia and iron deficiency in children with potential celiac disease. J Pediatr Gastroenterol Nutr 
2017; 64: 56-62

20. Nemeth E, Ganz T. Anemia of inflammation. Hematol Oncol Clin North Am 2014; 28: $671-81$

21. Hadjivassiliou M, Mäki M, Sanders DS, Williamson CA, Grünewald RA, Woodroofe NM, et al. Autoantibody targeting of brain and intestinal transglutaminase in gluten ataxia. Neurology 2006; 66: 373-7

22. Riches PL, McRorie E, Fraser WD, Determann C, van't Hof R, Ralston SH. Osteoporosis associated with neutralizing autoantibodies against osteoprotegerin. $N$ Engl J Med 2009; 361: 1459-65

23. Hervonen K, Salmi TT, Kurppa K, Kaukinen K, Collin P, Reunala T. Dermatitis herpetiformis in children: a long-term follow-up study. Br J Dermatol 2014; 171: $1242-3$

24. Khatib M, Baker RD, Ly EK, Kozielski R, Baker SS. Presenting pattern of pediatric celiac disease. J Pediatr Gastroenterol Nutr 2016; 62: 60-3

25. Lubrano E, Ciacci C, Ames PR, Mazzacca G, Oriente P, Scarpa R. The arthritis of coeliac disease: prevalence and pattern in 200 adult patients. Br J Rheumatol 1996; 35: $1314-8$

26. Äärelä L, Nurminen S, Kivelä L, Huhtala H, Mäki M, Viitasalo A, et al. Prevalence and associated factors of abnormal liver values in children with celiac disease. Dig Liver Dis 2016; 48: 1023-9

27. Vajro P, Paolella G, Maggiore G, Giordano G. Pediatric celiac disease, cryptogenic hypertransaminasemia, and autoimmune hepatitis. J Pediatr Gastroenterol Nutr 2013; 56: $663-70$

28. Cheng J, Malahias T, Brar P, Minaya MT, Green PH. The association between celiac disease, dental enamel defects, and aphthous ulcers in a United States cohort. J Clin 
Gastroenterol 2010; 44: 191-4

29. Aloi M, Cucchiara S. Extradigestive manifestations of IBD in pediatrics. Eur Rev Med Pharmacol Sci 2009; 13: 23-32

30. Wacklin P, Kaukinen K, Tuovinen E, Collin P, Lindfors K, Partanen J, et al. The duodenal microbiota composition of adult celiac disease patients is associated with the clinical manifestation of the disease. Inflamm Bowel Dis 2013; 19: 934-41 
Figure legend

Figure 1. Severity of small-bowel mucosal villous atrophy at diagnosis (A) and adherence to gluten free diet (B) in 511 children with celiac disease divided into three subgroups according to the main clinical presentation at the time of diagnosis. 
A

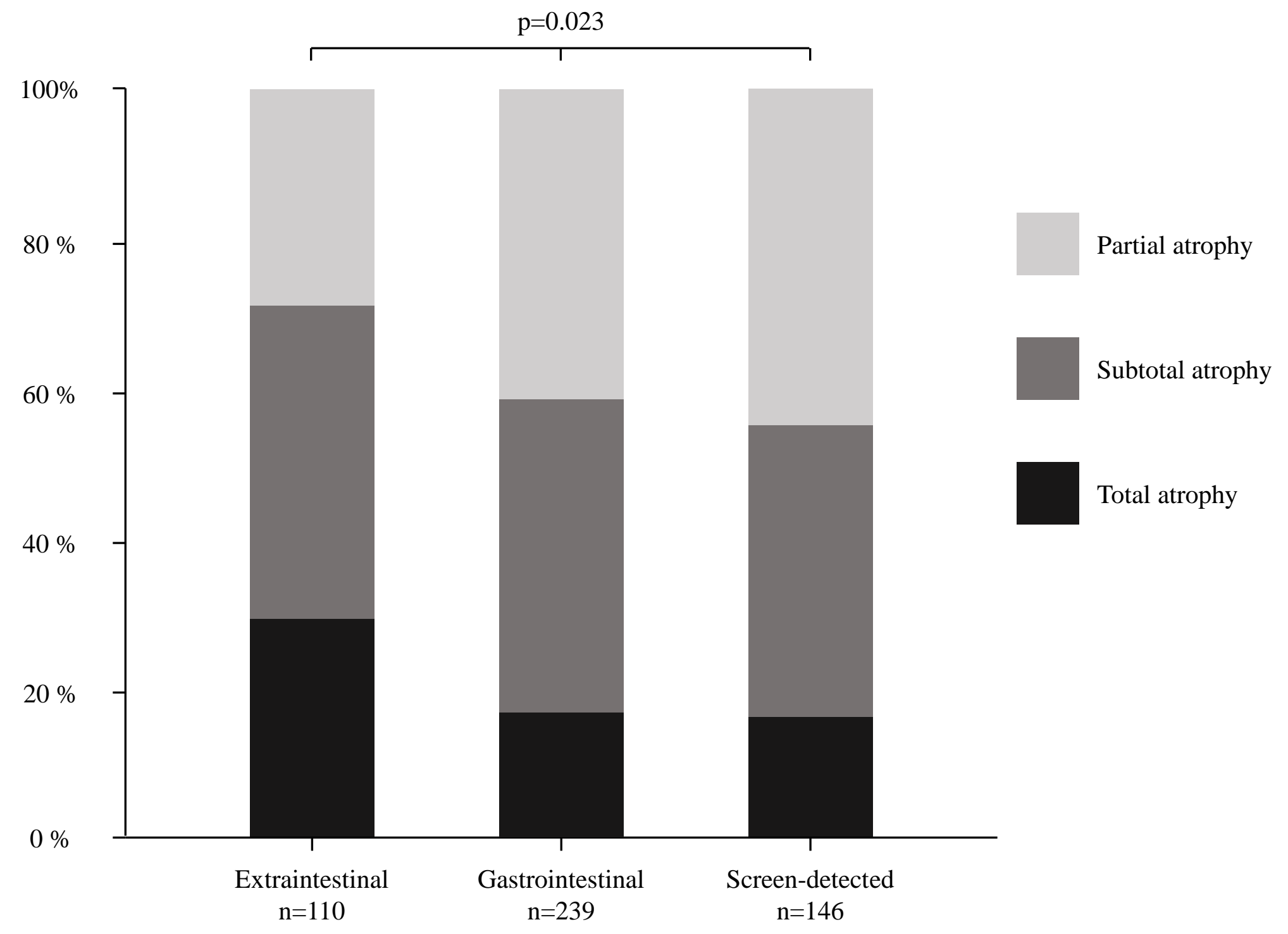


B

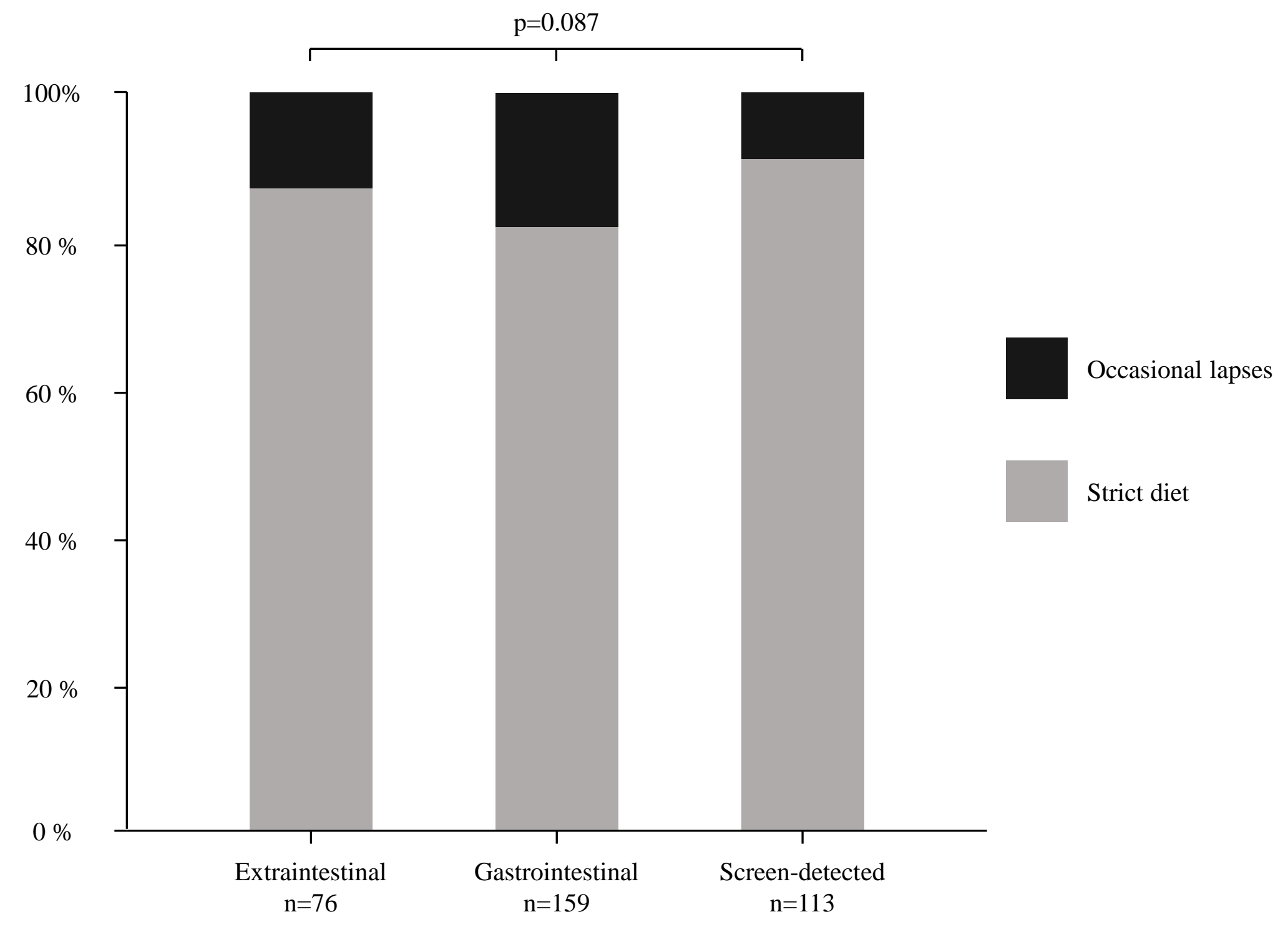


Table 1. Demographic data and prevalence of extraintestinal manifestations at the time of diagnosis in children with celiac disease, inflammatory bowel disease and functional gastrointestinal disorder

\begin{tabular}{|c|c|c|c|c|c|}
\hline & $\mathrm{n}$ & $\begin{array}{c}\text { Celiac disease } \\
n=511\end{array}$ & $n$ & $\begin{array}{l}\text { Functional disorder } \\
n=180 \\
\end{array}$ & $P$ value \\
\hline Age, median (quartiles), years & 511 & $7.6(4.8,11.6)$ & 180 & $10.6(6.8,13.7)$ & $<0.001$ \\
\hline Girls, \% & 511 & 65 & 179 & 53 & 0.003 \\
\hline \multicolumn{6}{|l|}{ Extraintestinal manifestations, \% } \\
\hline Skin symptoms ${ }^{1}$ & 511 & 15 & 180 & 4 & $<0.001$ \\
\hline Anemia & 459 & 18 & 103 & 5 & 0.001 \\
\hline Poor growth & 511 & 27 & 180 & 9 & 0.000 \\
\hline Enamel defects & 511 & 1 & 180 & 0 & 0.303 \\
\hline Neurological symptoms² & 511 & 9 & 180 & 3 & 0.006 \\
\hline Joint symptoms & 511 & 6 & 180 & 2 & 0.016 \\
\hline Recurrent aphtous ulcers & 511 & 2 & 180 & 3 & 0.476 \\
\hline Hypertransaminasemia & 152 & 9 & 63 & 3 & 0.159 \\
\hline Fatigue & 511 & 8 & 180 & 3 & 0.022 \\
\hline Other ${ }^{3}$ & 511 & 5 & 180 & 12 & 0.001 \\
\hline Total $^{4}$ & 511 & 62 & 180 & 33 & $<0.001$ \\
\hline
\end{tabular}

${ }^{1}$ Dermatitis herpetiformis, atopic dermatitis, acanthosis nigricans, undefined rash

${ }^{2}$ Headache, worsening of epilepsy or migraine, attention deficit/hyperactivity disorder-type symptoms, learning or communication difficulties, motor disorders

${ }^{3}$ Mouth/tongue pain, brittle nails, alocipea, epistaxis, shortness of breath, osteopenia, depression

${ }^{4}$ One or more extraintestinal symptom 
Table 2. Prevalence of extraintestinal manifestations at the time of diagnosis in 511 children with celiac disease divided according to the main clinical presentation.

\begin{tabular}{|c|c|c|c|c|c|c|c|}
\hline \multirow[b]{3}{*}{ Extraintestinal manifestation } & \multicolumn{6}{|c|}{ Main mode of presentation at diagnosis } & \multirow{3}{*}{ P value } \\
\hline & \multirow[b]{2}{*}{$\mathrm{n}$} & $\begin{array}{c}\text { Extraintestinal } \\
n=116\end{array}$ & & $\begin{array}{c}\text { Gastrointestinal } \\
n=249\end{array}$ & & $\begin{array}{c}\text { Screen-detected } \\
n=146\end{array}$ & \\
\hline & & $\%$ & $\mathrm{n}$ & $\%$ & $\mathrm{n}$ & $\%$ & \\
\hline Skin symptoms ${ }^{1}$ & 116 & 19 & 249 & 15 & 146 & 13 & 0.400 \\
\hline Anemia & 105 & 35 & 213 & 16 & 141 & 7 & $<0.001$ \\
\hline Poor growth & 116 & 56 & 249 & 21 & 146 & 14 & $<0.001$ \\
\hline Enamel defects & 116 & 1 & 249 & 1 & 146 & 0 & 0.605 \\
\hline Neurological symptoms ${ }^{2}$ & 116 & 10 & 249 & 10 & 146 & 8 & 0.763 \\
\hline Joint symptoms & 116 & 15 & 249 & 5 & 146 & 1 & $<0.001$ \\
\hline Aphtous ulcers & 116 & 4 & 249 & 2 & 146 & 1 & 0.143 \\
\hline Hypertransaminasemia & 46 & 13 & 80 & 6 & 26 & 8 & 0.421 \\
\hline Fatigue & 116 & 16 & 249 & 9 & 146 & 1 & $<0.001$ \\
\hline Other $^{3}$ & 116 & 10 & 249 & 4 & 146 & 2 & 0.014 \\
\hline Total $^{4}$ & 116 & 100 & 249 & 60 & 146 & 37 & $<0.001$ \\
\hline
\end{tabular}

${ }^{1}$ Dermatitis herpetiformis, atopic dermatitis, acanthosis nigricans, undefined rash

${ }^{2}$ Headache, worsening of epilepsy or migraine, attention deficit/hyperactivity disorder-type symptoms, learning or communication difficulties, motor disorders

${ }^{3}$ Mouth/tongue pain, brittle nails, alocipea, epistaxis, shortness of breath, osteopenia, depression

${ }^{4}$ One or more extraintestinal symptom 
Table 3. Age, growth data, serum autoantibodies and selected laboratory values at the time of diagnosis in 511 children with celiac disease divided according to the main clinical presentation.

\begin{tabular}{|c|c|c|c|c|c|c|c|}
\hline & \multicolumn{2}{|r|}{$\begin{array}{c}\text { Extraintestinal } \\
\quad \mathrm{n}=116\end{array}$} & \multicolumn{2}{|r|}{$\begin{array}{c}\text { Gastrointestinal } \\
n=249\end{array}$} & \multicolumn{2}{|r|}{$\begin{array}{c}\text { Screen-detected } \\
\mathrm{n}=146\end{array}$} & \multirow[b]{2}{*}{$P$ value } \\
\hline & $\mathrm{n}^{1}$ & Median $\left(\mathrm{Q}_{1}, \mathrm{Q}_{3}\right)$ & $n^{1}$ & $\operatorname{Median}\left(\mathrm{Q}_{1}, \mathrm{Q}_{3}\right)$ & $\mathrm{n}^{1}$ & $\operatorname{Median}\left(\mathrm{Q}_{1}, \mathrm{Q}_{3}\right)$ & \\
\hline Age, years & 116 & $10.0(4.6,12.3)$ & 249 & $7.4(5.0,11.0)$ & 146 & $7.0(4.1,11.6)$ & 0.092 \\
\hline Height, SD & 63 & $-0.2(-1.0,0.7)$ & 110 & $0.1(-0.7,1.0)$ & 87 & $0.3(-0.5,1.2)$ & 0.173 \\
\hline Body mass index, $\mathrm{kg} / \mathrm{m}^{2}$ & 45 & $16.4(14.9,18.6)$ & 90 & $16.3(15.0,18.5)$ & 67 & $16.3(15.0,18.0)$ & 0.891 \\
\hline EmA, titer & 79 & $1: 500(1: 100,1: 2000)$ & 159 & $1: 500(1: 100,1: 1000)$ & 97 & $1: 500(1: 100,1: 2000)$ & 0.146 \\
\hline TG2ab, U/l & 38 & $51(14,80)$ & 116 & $43(17,80)$ & 80 & $65(25,115)$ & 0.030 \\
\hline Hemoglobin, g/l & 87 & $124(105,131)$ & 174 & $123(114,131)$ & 81 & $126(121,135)$ & 0.032 \\
\hline Ferritin, $\mu \mathrm{g} / \mathrm{l}$ & 32 & $12.0(7.0,23.0)$ & 52 & $13.0(6.3,22.8)$ & 25 & $10.0(6.0,17.0)$ & 0.725 \\
\hline Transferrin receptor $1, \mathrm{mg} / \mathrm{l}$ & 27 & $4.4(3.5,7.7)$ & 41 & $4.2(3.4,5.7)$ & 18 & $4.5(3.1,6.1)$ & 0.604 \\
\hline Albumin, g/l & 23 & $38.0(37.0,41.0)$ & 52 & $38.0(35.5,40.0)$ & 19 & $41.0(38.0,42.0)$ & 0.051 \\
\hline Alkaline phosphatase, U/l & 31 & $179(136,245)$ & 56 & $212(168,238)$ & 19 & $194(161,226)$ & 0.497 \\
\hline Alanine aminotransferase, $\mathrm{U} / \mathrm{l}$ & 46 & $21.5(16.0,30.3)$ & 79 & $20.0(15.0,24.0)$ & 25 & $20.0(16.0,25.50)$ & 0.292 \\
\hline $\mathrm{TSH}, \mathrm{mU} / \mathrm{l}$ & 48 & $2.7(1.7,3.7)$ & 74 & $2.4(1.6,3.1)$ & 41 & $2.0(1.5,3.2)$ & 0.138 \\
\hline Thyroxine, pmol/l & 29 & $14.4(12.6,16.2)$ & 26 & $14.7(13.1,16.2)$ & 10 & $15.2(14.0,18.0)$ & 0.605 \\
\hline
\end{tabular}

$\mathrm{Q}_{1}, \mathrm{Q}_{3}=$ lower and upper quartiles; EmA = endomysial antibodies; $\mathrm{TG} 2 \mathrm{ab}=$ transglutaminase 2 antibodies; $\mathrm{TSH}=$ thyroid stimulating hormone ${ }^{1}$ Data available 